\title{
Application of Genome Editing Techniques in Immunology
}

\author{
Agata O. Zych ${ }^{1,2} \cdot$ Malgorzata Bajor $^{1} \cdot$ Radoslaw Zagozdzon $^{3,4,5}$
}

Received: 12 June 2017 / Accepted: 6 January 2018 / Published online: 17 January 2018

(c) The Author(s) 2018. This article is an open access publication

\begin{abstract}
The idea of using the effector immune cells to specifically fight cancer has recently evolved into an exciting concept of adoptive cell therapies. Indeed, genetically engineered $\mathrm{T}$ cells expressing on their surface recombinant, cancer-targeted receptors have been shown to induce promising response in oncological patients. However, in addition to exogenous expression of such receptors, there is also a need for disruption of certain genes in the immune cells to achieve more potent disease-targeted actions, to produce universal chimeric antigen receptor-based therapies or to study the signaling pathways in detail. In this review, we present novel genetic engineering methods, mainly TALEN and CRISPR/Cas9 systems, that can be used for such purposes. These unique techniques may contribute to creating more successful immune therapies against cancer or prospectively other diseases as well.
\end{abstract}

Keywords Adoptive therapy · Cancer · Viral diseases · Immunotherapy · TALEN · CRISPR/Cas $9 \cdot$ Genome editing

\section{Introduction}

The fundamental concept of adoptive cell therapies (ACT) against cancer or viral diseases is that immune effector cells can be isolated, expanded and returned to the patient to achieve a potent and disease-targeted cytotoxic activity (reviewed in Perica et al. 2015). For decades, however, ACT have been bringing only a modest success, as the classical recognition of target cells via endogenous T-cell receptor (TCR) is often inefficient for the cure (Dudley et al. 2008). This situation has been dramatically changed following introduction of genetic modifications of the effector cells that redirect them to target a chosen antigen (Fujiwara 2014). These modifications are usually following one out

Radoslaw Zagozdzon

radoslaw.zagozdzon@wum.edu.pl

1 Department of Immunology, Medical University of Warsaw, Warsaw, Poland

2 Postgraduate School of Molecular Medicine, Medical University of Warsaw, Warsaw, Poland

3 Department of Immunology, Transplantology and Internal Medicine, Medical University of Warsaw, Warsaw, Poland

4 Institute of Biochemistry and Biophysics, Polish Academy of Sciences, Warsaw, Poland

5 Department of Clinical Immunology, Medical University of Warsaw, Nowogrodzka 59, 02-006 Warsaw, Poland of two main streams: (1) introduction of recombinant $\alpha$ and $\beta$ chains pairing into exogenous tumor-specific TCR or (2) introduction of a chimeric antigen receptor (CAR) targeting a chosen surface molecule on cancer cells.

Although much improved, the immune effector cells expressing exogenous cancer-specific receptors still face considerable limitations, mainly due to three types of factors. The first is their susceptibility to inhibition via the natural immune checkpoint signaling, e.g. the programmed death-1 (PD-1)-mediated route (John et al. 2013). The second factor is related to the presence of the endogenous TCR, that following activation of the effector cell can potentially mediate severe autoimmune complications of the autologous T-cell transplant or graft-versus-host disease in allogeneic settings. Also in this context, the heterologous pairing of the $\alpha$ and $\beta$ chains of recombinant TCR with respective chains of endogenous TCR chains may attenuate their antigenic specificity or lead to autoreactivity (Heemskerk et al. 2007; van Loenen et al. 2010). Finally, the presence of intrinsic MHC class I molecules on the effector cells prevents their application in allogeneic settings as the off-the-shelf ACT, which makes the adoptive therapies considerably more expensive. To overcome these limitations, genome editing methods have been recently employed (Provasi et al. 2012).

Targeted genome editing (reviewed in Guha et al. 2017) constitutes a powerful tool for biological research and potential approach for genetic therapy. The most general concept 
behind genome editing is the introduction of double-strand breaks within DNA sequence in a region of interest, followed by an action of endogenous repair machinery to induce targeted mutations. The changes in the DNA structure can be repaired by two broad mechanisms: error-prone non-homologous end joining (NHEJ) or homology directed repair (HDR). In case of lack of a homologous repair template, the NHEJ may lead to insertion/deletion (in/dels) events, and thus cause changes in the open reading frame of the target gene (Martins-Rocha et al. 2015).

Lately, the most commonly used tools in genetic engineering are meganucleases (MN), zinc-finger nucleases (ZFN), transcription activator-like effector nucleases (TALEN) and clustered regularly interspaced short palindromic repeats (CRISPR) complexes, with the substantial predominance of the last technique in recent years. The main advantages and shortcomings of gene editing methods are summarized in Table 1.

\section{Meganucleases}

Meganucleases, also called homing endonucleases, are an engineered version of naturally occurring endonucleases, which are able to recognize and cleave considerably large
DNA sequences ( 14-40 bps) very rare in the most genomes (Stoddard 2011). Recognition of the unique sequences makes MN a very specific, non-toxic and highly suitable tool for genome engineering. However, the insufficiency of naturally occurring $\mathrm{MN}$ and limited variety of recognized sequences constitute the main drawbacks of this method. Moreover, the recognition and cleavage functions of MN are encoded in a single domain where the part of their structure is involved in a complex system of DNA interactions. The intricacy of the desired targeted sequence design has been partially solved by few scientific groups using fusion chimeras or mutating specific residues in the DNA binding scaffold (Silva et al. 2011; Zaslavskiy et al. 2014). Additionally, various companies managed to develop procedures to modify MN for use in genome editing to induce targeted recombination and correction of the RAG1 gene related to severe combined immunodeficiency (SCID) (Grizot et al. 2009) or XPC gene associated with xeroderma pigmentosum in skin cells (Arnould et al. 2007). A recently published study has shown a successful application of meganucleasemediated TCR $\alpha$-chain knock-out under conditions for optimal T-cell stimulation (MacLeod et al. 2017). Nonetheless, the procedure of "programming" $\mathrm{MN}$ to recognize the given sequence requires specialized knowledge and technology, and makes this approach extremely laborious. Due to the

Table 1 Comparison of the main genome editing methods

\begin{tabular}{|c|c|c|c|}
\hline Method & Advantages & Disadvantages & Limitations \\
\hline $\mathrm{MN}$ & $\begin{array}{l}\text { High specificity } \\
\text { Low toxicity } \\
\text { Recognition of large DNA sequences }\end{array}$ & $\begin{array}{l}\text { Extremely laborious } \\
\text { Single domain encoding two important } \\
\text { MN functions: recognition and cleav- } \\
\text { age }\end{array}$ & $\begin{array}{l}\text { Insufficient variety of recognized } \\
\text { sequences }\end{array}$ \\
\hline $\mathrm{ZFN}$ & $\begin{array}{l}\text { Recognition of any sequence } \\
\text { High efficiency }\end{array}$ & $\begin{array}{l}\text { High cost } \\
\text { Complexity of protein domains } \\
\text { Pairs of ZFNs are required to target any } \\
\text { specific locus } \\
\text { Requires screening to detect targeted } \\
\text { events in animals } \\
\text { Off-target effects }\end{array}$ & ZFN recognizes 3-6 nucleotide sequences \\
\hline TALEN & $\begin{array}{l}\text { TALE monomer recognizes single } \\
\text { nucleotide in target sequence } \\
\text { Lower cost than ZFN } \\
\text { High specificity }\end{array}$ & $\begin{array}{l}\text { Identical repeat sequences within TALE } \\
\text { array - cloning challenge } \\
\text { Complexity of protein domains } \\
\text { Large size of TALE molecules difficult } \\
\text { to deliver to the cells } \\
\text { Pairs of TALENs are required to target } \\
\text { any specific locus }\end{array}$ & $\begin{array}{l}\text { Binding efficiency depends on the pres- } \\
\text { ence of thymidine nucleotide before the } \\
5 \text { ' end of a sequence }\end{array}$ \\
\hline CRISPR/Cas9 & $\begin{array}{l}\text { Simplicity } \\
\text { Efficiency } \\
\text { Low cost } \\
\text { High precision } \\
\text { Versatility } \\
\text { Multiplexed mutations } \\
\text { Ability to obtain mutant organism in one } \\
\text { generation }\end{array}$ & $\begin{array}{l}\text { High possibility of off-target effects } \\
\text { Mosaicism }\end{array}$ & $\begin{array}{l}\text { Limited target sequences due to necessity } \\
\text { of presence of PAM sequences }\end{array}$ \\
\hline
\end{tabular}

$M N$ meganucleases, ZFN zinc-finger nucleases, TALEN transcription activator-like effector nucleases, CRISPR clustered regularly interspaced short palindromic repeats, $P A M$ proto-spacer-adjacent motifs 
fact that meganucleases are very difficult to optimize to target specific sequence, MN have not been widely used for genome engineering.

\section{Zinc-Finger Nucleases}

ZFN are fusion proteins with engineered DNA binding domains and a non-specific nuclease domain from the FokI restriction enzyme. ZFN were the first reagents utilized to introduce targeted changes into the genome (Durai et al. 2005). Individual ZF motif consists of approximately 30 amino acids organized in a conserved $\beta \beta \alpha$ structure stabilized by the hydrophobic cluster of residues and chelation of the zinc ion. The DNA binding is performed by interaction of several amino acids of the ZF $\alpha$-helix with three base pairs in the major groove of DNA (Gaj et al. 2013). Typically, each ZFN recognizes 3-6-nucleotide sequences. ZF motifs can be designed to recognize almost any DNA sequence. Nucleases attached to ZF work as dimers, thus pairs of ZFN are required to target any specific locus (Durai et al. 2005). Despite a theoretical possibility to target any specific sequence, ZFN approach has in fact a number of major disadvantages. Primarily, the high cost and complexity of protein domains design make this method unattractive. Furthermore, single nucleotide substitutions or improper interactions between domains increase the probability of inaccurate cleavage of target sequence (Nemudryi et al. 2014). Nevertheless, ZFN can potentially be of use for editing the genome of $\mathrm{T}$ cells mainly in HIV-related research (Perez et al. 2008), especially when combined with adenoassociated virus vectors to function as homology donors (Wang et al. 2016). ZFN approach was also used to mediate site-specific integration of therapeutic transgenes in hepatocytes within albumin gene. Expression of human factors VIII and IX were obtained in mouse models of hemophilia A and $\mathrm{B}$ at therapeutic levels as well as lysosomal enzymes that are deficient in Fabry and Gaucher diseases and in Hurler and Hunter syndromes (Sharma et al. 2015). What is more, ZFN technology have been successfully used to disrupt CCR5 gene in hematopoietic stem/progenitor cells (HSPC) (DiGiusto et al. 2016). Currently there are several ongoing clinical studies utilizing ZFN approach against HIV-1 infection, hemophilia B or mucopolysaccharidosis I/II (see Table 2).

\section{Transcription Activator-Like Effector Nucleases}

The method that was considered to overcome the ZFN drawbacks was TALEN (Fig. 1). Similarly to ZFN, the DNA binding domain that is fused with FokI enzyme in TALEN structure consists of a sequence of protein monomers (called
TALE). Unlike ZFN, a single TALE monomer binds to one nucleotide in the target sequence. The ability of TALEN method to recognize single bases is an unquestionable advantage in targeting desirable sequence in contrast to ZFN approach which recognizes nucleotide triplets. Each TALE monomer is composed of a series of 33-35 amino acid repeat domains. The two highly variable amino acid residues located at positions 12 and 13 (called repeat variable diresidue) are responsible for TALE specificity (Gaj et al. 2013; Joung and Sander 2013; Nemudryi et al. 2014). In TALEN, TALE monomers can be arbitrarily linked together to recognize the desired DNA sequence. However, due to the expanded identical repeat sequences, cloning of TALE arrays causes a major technical challenge (Christian et al. 2010; Miller et al. 2011). Furthermore, the critical point for binding efficiency is the presence of thymidine nucleotide before the $5^{\prime}$ end of a sequence bound by TALE monomer (Lamb et al. 2013).

TALEN technology has been utilized in ACT strategies on numerous occasions. For instance, Poirot et al. (2015) have described the TALEN-mediated multiplex genomeedited manufacturing platform for universal T-cell-based immunotherapies. Based on a similar approach, a successful application of the TALEN-edited [by disruption of TCR $\alpha$ constant (TRAC) and CD52 genes] CAR-T cells targeting CD19 in two HLA-mismatched infants with relapsed refractory B-cell acute lymphoblastic leukemia has been recently reported (Qasim et al. 2017). TALEN strategy has also been used to inactivate the PD-1 molecule in tumor-reactive lymphocytes (Menger et al. 2016). Two upcoming clinical trials are going to use this methodology in treatment of female patients with human papillomavirus (HPV)-related cervical intraepithelial neoplasia (see Table 2).

\section{CRISPR/Cas9}

Elucidation of the role of identified clustered regularly interspaced short palindromic repeats found for the first time in Escherichia coli in 1987 (Ishino et al. 1987) have revolutionized the manipulation of DNA and introduction of site-specific mutations. CRISPR/Cas is an adaptive immune system (reviewed in Hryhorowicz et al. 2017) found in many bacteria and archaea, which enables effective defense against the invasion of bacteriophages or viruses. This immune system allows prokaryotes to "memorize" foreign DNA by incorporating its fragments into CRISPR arrays and ensures fast response to another infection in the future (Barrangou et al. 2007). The CRISPR array is organized by series of short (approx. 23-44 bp) sequences called spacers which are separated by highly conserved similarly sized sequences repeats. These spacers originate from viral or phage DNA and serve as a genetic memory of previous infections (Barrangou et al. 


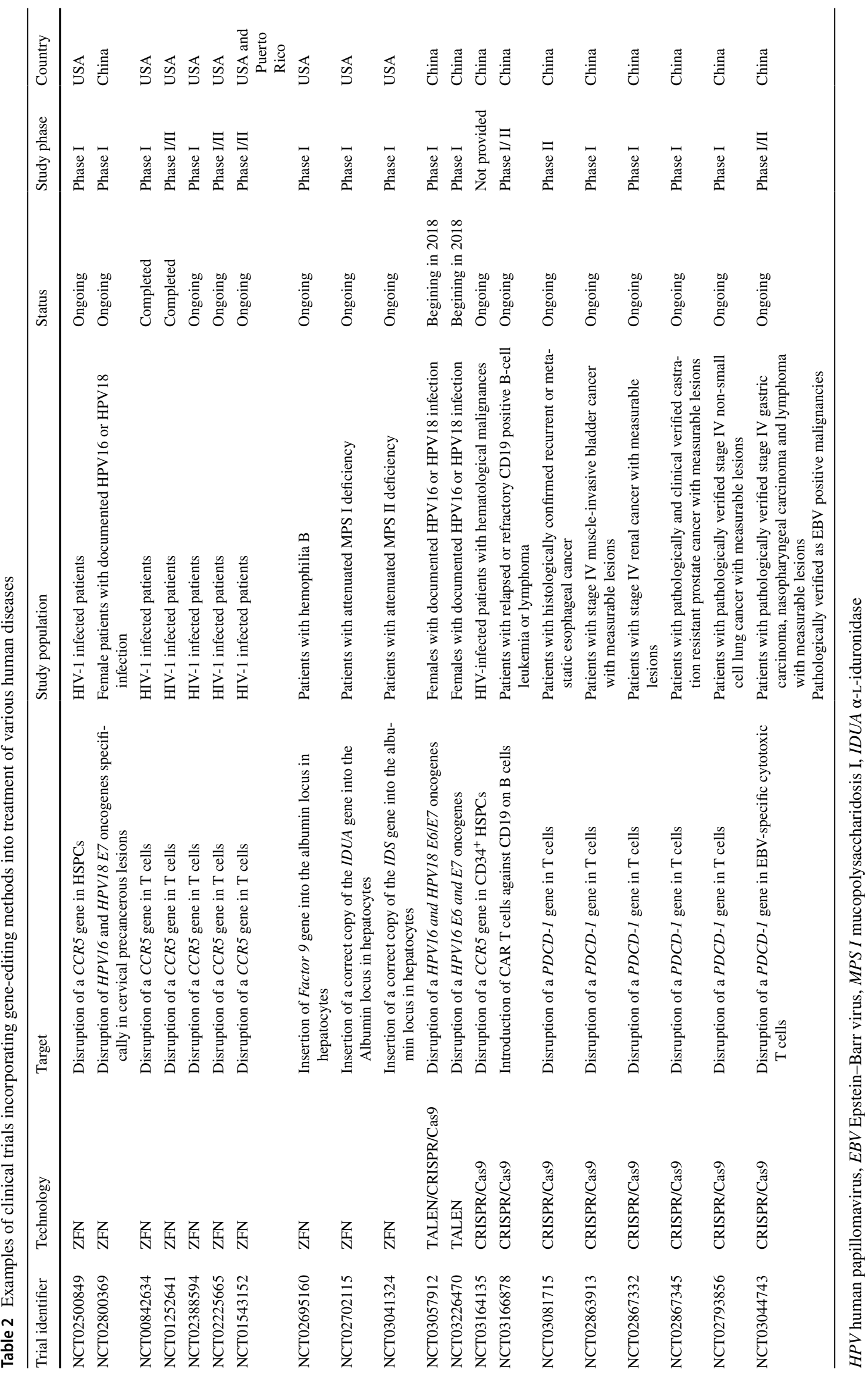


Fig. 1 Schematic representation of the double-strand DNA break introduction using TALEN.

FokI enzyme acts as a catalytic domain following the recognition of specific DNA sequences by TALEs (depicted as colorful rectangles)

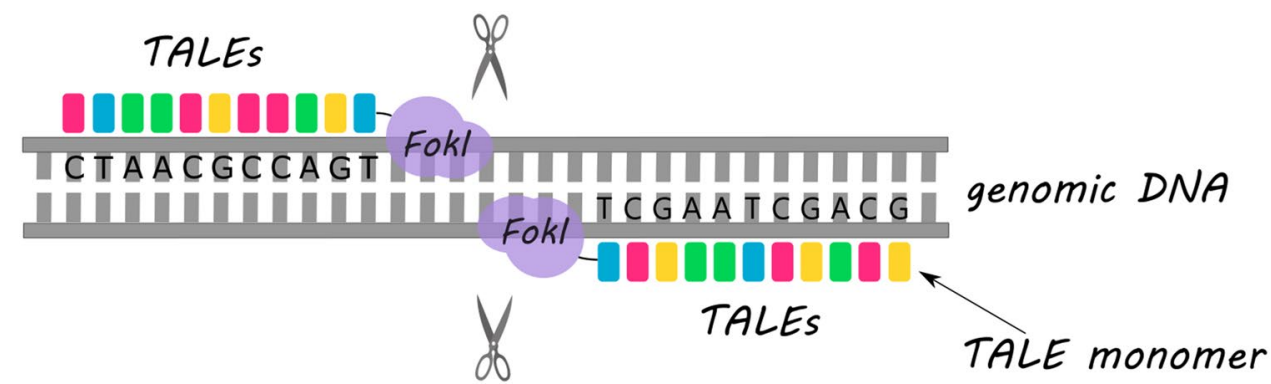

2007; Bolotin et al. 2005; Garneau et al. 2010; van der Ploeg 2009). Another very important compound of this system are Cas (CRISPR associated proteins) endonucleases, which mediate the double-strand breaks.

The CRISPR/Cas immune system performs its function in three general steps: adaptation, expression, and interference. During the first stage, short fragments of viral or phage DNA are incorporated into the CRISPR array. The integration of the new viral/phage DNA sequences is followed by duplication of a repeat, which in this way is forming a new spacer-repeat unit. Spacer precursors called proto-spacers are selected from invading DNA depending on the recognition of neighboring proto-spacer-adjacent motifs (PAM). PAM sequences are typically several nucleotides long and vary among different variants of the CRISPR/Cas system (Makarova et al. 2011). The arrangement of spacers within the CRISPR array corresponds to the sequence of invasion events. In the next stage, CRISPR array is transcribed and primary transcript pre-crRNA is produced, which then is processed to mature CRISPR RNA (crRNA) by RNase III. Depending on the CRISPR/Cas system class, this process can be mediated either by multiprotein CRISPR ribonucleoprotein complex or a single protein. In the last step-interference, crRNA directs Cas proteins to appropriate target within foreign DNA or RNA and Cas proteins perform cleavage of the invading genome (Terns and Terns 2013).

Despite the variety of the CRISPR/Cas systems in nature, the most commonly used type adapted to genome editing is class 2 type II CRISPR/Cas9. CRISPR/Cas9 requires two short RNA sequences: crRNA and transactivating crRNA (tracrRNA) to recognize and cleave foreign DNA sequences. During the action of Cas9 the crRNA hybridizes with the tracrRNA forming duplex crRNA:tracrRNA, which in the next step associates with Cas9. The crRNA is complementary to the target DNA sequence, while tracrRNA shows homology towards PAM and possess a binding site for the Cas9 which is indispensable for interference step (Karvelis et al. 2013). The Cas 9 comprises of two nuclease domains: $\mathrm{HNH}$ responsible for cleavage of the DNA strand complementary to the spacer sequence and RuvC that cleaves noncomplementary strand (Nishimasu et al. 2014). The most frequently commercially utilized version of CRISPR/Cas9 system consists of Cas9 protein from Streptococcus pyogenes and a chimeric single guide RNA (sgRNA), that is a fusion of crRNA and tracrRNA (Fig. 2). sgRNA can be designed to target any sequence followed by a $5^{\prime}-\mathrm{NGG}-3^{\prime}$ PAM sequence (Cong et al. 2013; Mali et al. 2013). Moreover, multiple genes can be targeted at the same time by introducing multiple sgRNAs at once (Cong et al. 2013). Despite the high efficiency, feasibility, and simplicity of target design CRISPR/Cas9 technique faces important complications. The most essential limitation of this method are mutations at sites with similar but not identical homology to the target sites (Cradick et al. 2013). To overcome random mutations, a number of modifications have been introduced to CRISPR/ Cas9 strategy, as described below.

One possibility involves shortening of sgRNA to create truncated sgRNA (trugRNA) to the length of less than 20 nucleotides (17-19) and this manipulation decreases undesired mutagenesis by 5000-fold without compromising the efficiency (Fu et al. 2014). The other option is to convert Cas9 nucleases into nickases that enhance genome editing specificity. Cas 9 nickases possess mutation in one of the endonuclease domains ( $\mathrm{RuvC}^{\mathrm{D} 10 \mathrm{~A}}$ or $\mathrm{HNH}^{\mathrm{H} 840 \mathrm{~A}}$ ) and hence cut only one strand of DNA generating single-strand breaks. Repair of individual nicks in the genome occur with high fidelity, without inducing in/dels, therefore, introduction of paired nicking can reduce unwanted off-target activity by 50-1000-fold (Chiang et al. 2016; Ran et al. 2013; Shen et al. 2014). Efficient reduction of in/dels caused by NHEJ mechanism was achieved also by inhibiting DNA ligase IV, a key enzyme in NHEJ pathway. This alteration showed great improvement in the efficiency of precise editing by CRISPR/Cas9 in fertilized zygotes and may be applicable also in other genetic engineering methods such as ZFN or TALEN (Maruyama et al. 2015).

Another way to reduce the occurrence of off-target mutations is generation of dimeric RNA-guided FokI nucleases (RFNs), that are able to recognize extended sequences and introduce modifications with high efficiencies. RFNs are created by fusing, wild-type FokI nuclease domain to catalytically inactive Cas9 (dCas9) protein. The FokI nuclease domain requires dimerization to perform DNA cleavage. Thus, it is highly unlikely that any 

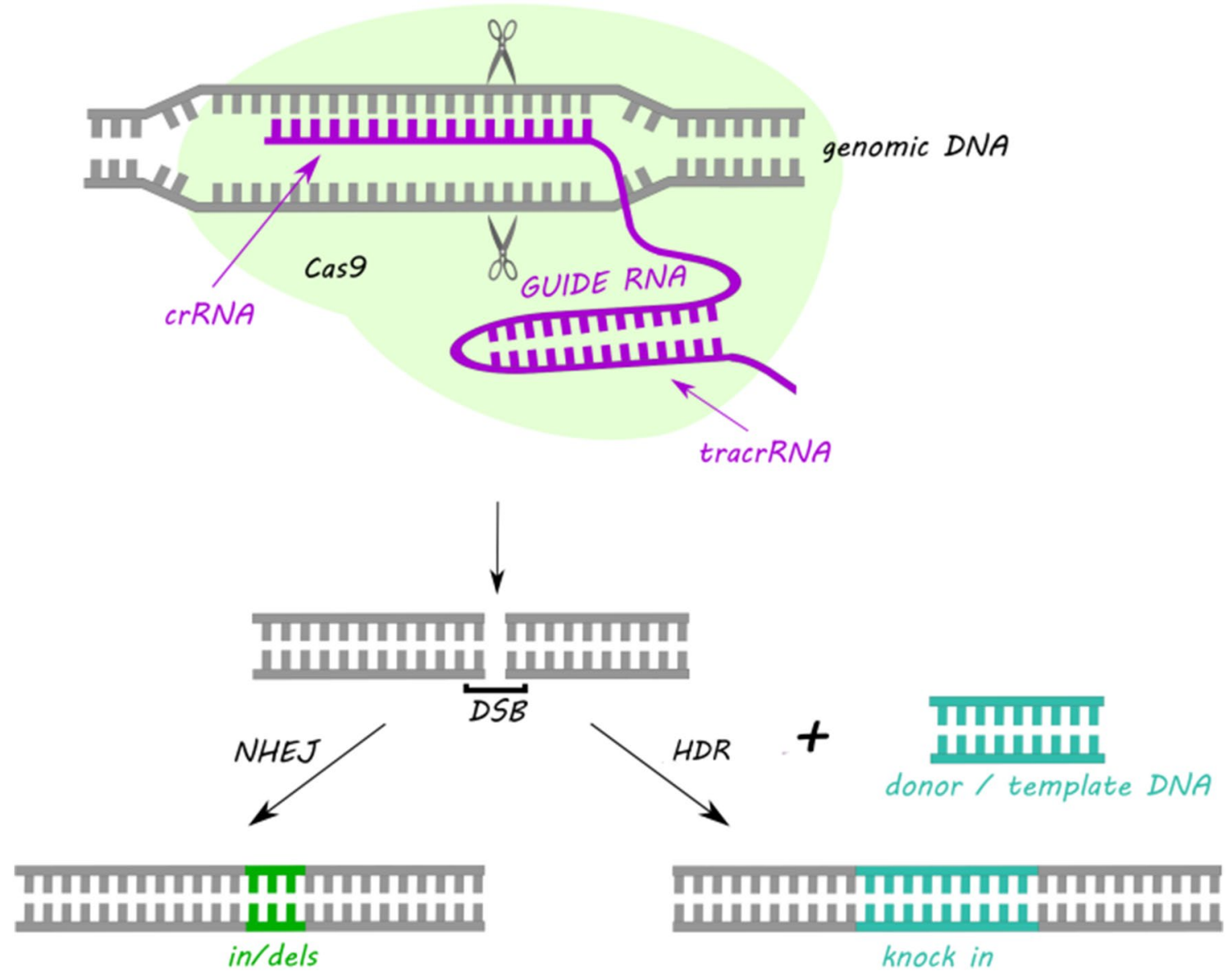

Fig. 2 Schematic representation of the double-strand DNA break introduction by a sgRNA-guided CRISPR/Cas9-based system and the main routes of DNA repairing. NHEJ non-homologous end joining, $D S B$ double-strand breaks, HDR homology directed repair

mutagenesis could be introduced at partially mismatched, off-target half-sites. Indeed, no detectable mutations were found in known off-target sites within targeted sequences with the use of RFN approach (Tsai et al. 2014). dCas9 alone enables targeting genomic DNA without cleaving it as a flexible and precise RNA-guided transcription regulation. This ability of dCas9 was applied in Cas9-SunTag system. SunTag is a repeating peptide array, which can recruit multiple copies of an antibody-fusion protein. In dCas9-SunTag were employed multiple VP64 domains (VP64-four copies of herpes virus transcriptional activation domain VP16) to a single dCas9 and this manipulation enhanced potently artificial activation of gene transcription without introducing genetic changes (Tanenbaum et al. 2014).

Apart from gene disruption and transcription activation, the CRISPR/Cas9 method enables introduction of a gene knock-in as well. The methodology is quite similar to gene knock-out and requires sgRNA, which targets the knock-in site, Cas9 protein and additionally a donor sequence of interest. In primary T cells, Cas9 ribonucleoproteins were used for targeted nucleotide replacement $C X C R 4$ and PDCD1 genes (Schumann et al. 2015).

With the emergence of CRISPR/Cas9 method, the modifications of hematopoietic progenitors cells or $\mathrm{T}$ cells have become easier to achieve. Indeed, numerous of such attempts have been made (Chi et al. 2016; Chu et al. 2016; Gundry et al. 2016; Gwiazda et al. 2016; Hendel et al. 2015; Li et al. 2015; Liu et al. 2017; Mandal et al. 2014; Schumann et al. 2015, Su et al. 2016, 2017), as exemplified in Table 3. Various clinically important genes were disrupted in human primary $\mathrm{CD}^{+}{ }^{+} \mathrm{T}$ cells and/or $\mathrm{CD} 34^{+}$hematopoietic and progenitor cells with high on-target efficacy and low incidence of off-target mutagenesis using CRISPR/Cas9 approaches e.g.: $\beta_{2}$-microglobulin (B2M) —encoding the accessory chain of major histocompatibility complex (MHC) class I (Liu et al. 2017; Mandal et al. 2014); chemokine receptor 5 (CCR5)-the main coreceptor used by CCR5-tropic HIV-1 strains (Mandal et al. 2014; Hendel et al. 2015; Li et al. 2015; Gwiazda et al. 2016); IL2RG-encoding common gamma chain of many interleukin receptors which mutations are responsible for SCID- $\mathrm{X}_{1}, \mathrm{HBB}$-encoding 
Table 3 Examples of genetic modifications of T lymphocytes or hematopoietic progenitor cells with the use of CRISPR/Cas9-based methods

\begin{tabular}{|c|c|c|c|}
\hline Modification/s & Targeted molecules & Cell type & References \\
\hline Gene disruption & B2M; CCR5 & $\begin{array}{l}\text { Human primary } \mathrm{CD} 4^{+} \mathrm{T} \text { cells } \\
\mathrm{CD} 34^{+} \text {hematopoietic progenitor cells }\end{array}$ & Mandal et al. (2014) \\
\hline Gene disruption & IL2RG, HBB and CCR5 & Human primary cells & Hendel et al. (2015) \\
\hline $\begin{array}{l}\text { Gene disruption } \\
\text { Knock-in }\end{array}$ & $\begin{array}{l}\text { CXCR4, PD1 } \\
12 \text { nucleotides (of CXCR4 or PD-1 gene) } \\
\text { with PAM sequence and restriction site for } \\
\text { Hind III }\end{array}$ & Human primary $\mathrm{CD}^{+} \mathrm{T}$ cells & Schumann et al. (2015) \\
\hline Gene disruption & CCR5 & Human primary $\mathrm{CD} 4^{+} \mathrm{T}$ cells & Li et al. (2015) \\
\hline Gene disruption & PD-1 & Human primary $\mathrm{T}$ cells & Su et al. (2016) \\
\hline Gene disruption & $\begin{array}{l}\text { Genes encoding B-cell or T-cell surface } \\
\text { markers (Rosa26, Prdm1, Ost4, Arf4, } \\
\text { Creld2, Zfp36, Edem1, Irf4, Myc, Xbp1, } \\
\text { Pou2af1) }\end{array}$ & Murine T cells and B cells & Chu et al. (2016) \\
\hline Gene disruption & Eed, Suz12, and DNMT3A & $\begin{array}{l}\text { Human and mouse hematopoietic progeni- } \\
\text { tor cells }\end{array}$ & Gundry et al. (2016) \\
\hline $\begin{array}{l}\text { Gene disruption } \\
\text { Upregulation of expression }\end{array}$ & $\begin{array}{l}\text { CD28 } \\
\text { CXCR4 }\end{array}$ & $\begin{array}{l}\text { JK28 cells } \\
\text { JS19 }\end{array}$ & Chi et al. (2016) \\
\hline Gene disruption & TCR $\alpha$, TIGIT, Lag3, Tim3 and CCR5 & Human primary $\mathrm{T}$ cells & Gwiazda et al. (2016) \\
\hline Gene disruption & PD-1 & $\mathrm{T}$ cells & Su et al. (2017) \\
\hline $\begin{array}{l}\text { Gene disruption } \\
\text { Knock-in }\end{array}$ & $\begin{array}{l}\text { TRAC, B2M, PD-1 } \\
\text { Anti-CD19 CAR }\end{array}$ & $\mathrm{T}$ cells & Liu et al. (2017) \\
\hline
\end{tabular}

$\beta$-globin, mutations within this gene cause sickle anemia and thalassemia (Hendel et al. 2015); CXCR4 — an $\alpha$ chemokine receptor, used by HIV virus to infect $\mathrm{T}$ cells (Schumann et al. 2015) and PDCD1, encoding PD-1 (Schumann et al. 2015, Su et al. 2016, 2017; Liu et al. 2017). Yet, the greatest interest is focused around introduction of CAR-T cells. The concept of making an adoptive immunotherapy accessible for every patient, even those without enough good quality $\mathrm{T}$ cells, is to develop an allogeneic adoptive transfer. This idea assumes creating universal CAR-T cells obtained from a healthy donor $\mathrm{T}$ cells and further application of modified $\mathrm{T}$ cells to multiple patients. As mentioned above, to succeed the endogenous $\alpha \beta$ TCR need to be disrupted as well as MHC class I to minimize their immunogenicity. Recent publication is showing a great potential of modified T cells by CRISPR/Cas9 in this context. Anti-CD19 CAR has already been reported to possess a potent anti-tumor activity in B-cell non-Hodgkin lymphoma, acute lymphoblastic leukemia or chronic lymphocytic leukemia patients (Turtle et al. 2016). Liu et al. (2017) obtained double (B2M, TRAC) or triple knock-outs (B2M, TRAC and PD-1) with the use of CRISPR/Cas9 technology and insertion of antiCD19 CAR in T cells. All variants were tested for off-target mutations and no significant differences between control cells and knock-out/knock-in cells were found. Double knock-out cells showed reduced proliferation as a result of loss of TCR function. Importantly, the cytotoxic functions remained unchanged in comparison to the standard CAR-T cells. Furthermore, in vivo experiment showed a maintained
CD19 anti-tumor specificity despite multiplex gene editing with CRISPR/Cas9 (Liu et al. 2017). CRISPR/Cas9 method shows great potential for genome editing even in modifications of T cells. Apart from the therapeutic use, CRISPR/ Cas 9 can also be utilized for studying the intracellular signaling in T cells (Chi et al. 2016).

In clinical settings, researchers in China have recently initiated a clinical trial to evaluate CAR-T cells modified by the CRISPR/Cas9 method (ClinicalTrials.gov Identifier: NCT03166878). They will combine the lentiviral delivery of anti-CD19 CAR and CRISPR RNA electroporation to disrupt endogenous TCR- and B2M-encoding genes simultaneously. Several other Chinese clinical trials are investigating the efficacy of CRISPR/Cas9-mediated PD-1 knock-out T cells in patients with malignancies such as: advanced esophageal carcinoma (NCT03081715), muscle-invasive bladder cancer (NCT02863913), metastatic renal cell carcinoma (NCT02867332) and others (see Table 2).

\section{Prospects of Gene Editing Methods in Treatment of Diseases}

Gene therapy holds promise of an attractive and expectantly precise treatment of variety of diseases in the future. Enabling treating illnesses caused by gene mutations by replacing DNA fragments with a correct copy of the gene or inactivating improperly functioning genes are widely studied subjects. Despite various drawbacks and limitations 
of gene editing methods (see Table 1), they have already been successfully used in a number of clinical studies (Table 2). These trials often reach beyond cancer treatment. For instance, several clinical studies are utilizing ZFN technology to disrupt CCR5 gene in HSPC or T cells which is required for HIV virus to enter into T cells (NCT02500849; NCT02388594; NCT02225665; NCT01543152). A number of similar clinical trials conducted earlier, have revealed that ZFN approach is mostly safe for application in humans (NCT00842634, NCT01044654). Generally, infusion of ZFN-modified autologous T cells was associated with mild side effects and only one serious side effect was observed in relation to transfusion. During those studies, a significant increase of $\mathrm{CD} 4^{+} \mathrm{T}$ cells was observed. What is more, HIV DNA decreased in most patients and HIV RNA was undetectable in one of four evaluated patients (Tebas et al. 2014). Those results give an encouraging starting point for the application of genetic engineering methods in treating various viral infections hampering functionality of the immune system, though this approach needs to be assessed in a wider group of patients. However, the complexity of ZFN and TALEN design may lead to more extensive development of simpler and more feasible ways to use CRISPR/ Cas9 method. Furthermore, safety issues and off-target effects are being solved by various modifications such as: Cas9 nickase, using Cas9 mRNA or adeno-associated vectors for introduction of system components into the cells with high efficiency and little or no risk for the patient. The interesting example is given by a recent publication of Yin et al. (2016), where researchers utilizing CRISPR/Cas9 system successfully restored the correct $F A H$ gene function in $6 \%$ of liver cells in a mouse model of tyrosinemia type I, which was enough to cure the disease. Thus, with emergence of easy, inexpensive and highly efficient CRISPR/ Cas9-based methodology, more and more clinical trials are testing safety of this approach in treating various cancers or viral infections (see Table 3). One must assume that in the near future additional genome editing-based therapies will be available to treat various somatic diseases. Obviously, appearance of gene editing methods creates a temptation to therapeutically modify human embryos, however, discussion of these strategies ranges beyond the scope of the current review.

\section{Conclusion}

In the last several years, we have observed a revolution in ACT used in oncology due to the capabilities of new methods for retargeting the immune effector cells against the cancer cells. Most recently, it has been increasingly clear that the gene editing techniques, such as TALEN or CRISPR/Cas9, may further refine ACT or direct genetic therapies to become a successful, universal and cost-effective strategy against cancer and perhaps a range of other diseases as well.

Acknowledgements This work was supported by European Commission Horizon 2020 Programme 692180-STREAM-H2020-TWINN-2015.

\section{Compliance with Ethical Standards}

Conflict of interest The authors declare that they have no conflict of interest.

Open Access This article is distributed under the terms of the Creative Commons Attribution 4.0 International License (http://creativecommons.org/licenses/by/4.0/), which permits unrestricted use, distribution, and reproduction in any medium, provided you give appropriate credit to the original author(s) and the source, provide a link to the Creative Commons license, and indicate if changes were made.

\section{References}

Arnould S, Perez C, Cabaniols JP et al (2007) Engineered I-CreI derivatives cleaving sequences from the human XPC gene can induce highly efficient gene correction in mammalian cells. J Mol Biol 371:49-65

Barrangou R, Fremaux C, Deveau H et al (2007) CRISPR provides acquired resistance against viruses in prokaryotes. Science 315:1709-1712

Bolotin A, Quinquis B, Sorokin A et al (2005) Clustered regularly interspaced short palindrome repeats (CRISPRs) have spacers of extrachromosomal origin. Microbiology 151(Pt 8):2551-2561

Chi S, Weiss A, Wang H (2016) A CRISPR-based toolbox for studying T cell signal transduction. Biomed Res Int 2016:5052369

Chiang TW, le Sage C, Larrieu D et al (2016) CRISPR-Cas9D10A nickase-based genotypic and phenotypic screening to enhance genome editing. Sci Rep 6:24356

Christian M, Cermak T, Doyle EL et al (2010) Targeting DNA doublestrand breaks with TAL effector nucleases. Genetics 186:757-761

Chu VT, Graf R, Wirtz T et al (2016) Efficient CRISPR-mediated mutagenesis in primary immune cells using CrispRGold and a C57BL/6 Cas9 transgenic mouse line. Proc Natl Acad Sci USA 113:12514-12519

Cong L, Ran FA, Cox D et al (2013) Multiplex genome engineering using CRISPR/Cas systems. Science 339:819-823

Cradick TJ, Fine EJ, Antico CJ et al (2013) CRISPR/Cas9 systems targeting $\beta$-globin and CCR5 genes have substantial off-target activity. Nucleic Acids Res 41:9584-9592

DiGiusto DL, Cannon PM, Holmes MC et al (2016) Preclinical development and qualification of ZFN-mediated CCR5 disruption in human hematopoietic stem/progenitor cells. Mol Ther Methods Clin Dev 3:16067

Dudley ME, Yang JC, Sherry R et al (2008) Adoptive cell therapy for patients with metastatic melanoma: evaluation of intensive myeloablative chemoradiation preparative regimens. J Clin Oncol 26:5233-5239

Durai S, Mani M, Kandavelou K et al (2005) Zinc finger nucleases: custom-designed molecular scissors for genome engineering of plant and mammalian cells. Nucleic Acids Res 33:5978-5990 
Fu Y, Sander JD, Reyon D et al (2014) Improving CRISPR-Cas nuclease specificity using truncated guide RNAs. Nat Biotechnol $32: 279-284$

Fujiwara H (2014) Adoptive immunotherapy for hematological malignancies using $\mathrm{T}$ cells gene-modified to express tumor antigenspecific receptors. Pharmaceuticals 7:1049-1068

Gaj T, Gersbach CA, Barbas CF (2013) ZFN, TALEN and CRISPR/ Cas-based methods for genome engineering. Trends Biotechnol 31:397-405

Garneau JE, Dupuis M, Villion M et al (2010) The CRISPR/Cas bacterial immune system cleaves bacteriophage and plasmid DNA. Nature 468:67-71

Grizot S, Smith J, Daboussi F et al (2009) Efficient targeting of a SCID gene by an engineered single-chain homing endonuclease. Nucleic Acids Res 37:5405-5419

Guha TK, Wai A, Hausner G (2017) Programmable genome editing tools and their regulation for efficient genome engineering. Comput Struct Biotechnol J 15:146-160

Gundry MC, Brunetti L, Lin A et al (2016) Highly efficient genome editing of murine and human hematopoietic progenitor cells by CRISPR/Cas9. Cell Rep 17:1453-1461

Gwiazda KS, Grier AE, Sahni J et al (2016) High efficiency CRISPR/ Cas9-mediated gene editing in primary human T-cells using mutant adenoviral E4orf6/E1b55k "helper" proteins. Mol Ther 24:1570-1580

Heemskerk MH, Hagedoorn RS, Hoorn MA et al (2007) Efficiency of $\mathrm{T}$-cell receptor expression in dual-specific $\mathrm{T}$ cells is controlled by the intrinsic qualities of the TCR chains within the TCR-CD3 complex. Blood 109:235-243

Hendel A, Bak RO, Clark JT et al (2015) Chemically modified guide RNAs enhance CRISPR-Cas genome editing in human primary cells. Nat Biotechnol 33:985-989

Hryhorowicz M, Lipiński D, Zeyland J et al (2017) CRISPR/Cas9 immune system as a tool for genome engineering. Arch Immunol Ther Exp 65:233-240

Ishino Y, Shinagawa H, Makino K et al (1987) Nucleotide sequence of the iap gene, responsible for alkaline phosphatase isozyme conversion in Escherichia coli, and identification of the gene product. J Bacteriol 169:5429-5433

John LB, Devaud C, Duong CP et al (2013) Anti-PD-1 antibody therapy potently enhances the eradication of established tumors by gene-modified T cells. Clin Cancer Res 19:5636-5646

Joung JK, Sander JD (2013) TALENs: a widely applicable technology for targeted genome editing. Nat Rev Mol Cell Biol $14: 49-55$

Karvelis T, Gasiunas G, Miksys A et al (2013) crRNA and tracrRNA guide Cas9-mediated DNA interference in Streptococcus thermophilus. RNA Biol 10:841-851

Lamb BM, Mercer AC, Barbas CF (2013) Directed evolution of the TALE N-terminal domain for recognition of all $5^{\prime}$ bases. Nucleic Acids Res 41:9779-9785

Li C, Guan X, Du T et al (2015) Inhibition of HIV-1 infection of primary CD4 + T-cells by gene editing of CCR5 using adenovirusdelivered CRISPR/Cas9. J Gen Virol 96:2381-2393

Liu X, Zhang Y, Cheng C et al (2017) CRISPR-Cas9-mediated multiplex gene editing in CAR-T cells. Cell Res 27:154-157

MacLeod DT, Antony J, Martin AJ et al (2017) Integration of a CD19 CAR into the TCR alpha chain locus streamlines production of allogeneic gene-edited CAR T cells. Mol Ther 25:949-961

Makarova KS, Haft DH, Barrangou R et al (2011) Evolution and classification of the CRISPR-Cas systems. Nat Rev Microbiol 9:467-477

Mali P, Yang L, Esvelt KM et al (2013) RNA-guided human genome engineering via Cas9. Science 339:823-826
Mandal PK, Ferreira LM, Collins R et al (2014) Efficient ablation of genes in human hematopoietic stem and effector cells using CRISPR/Cas9. Cell Stem Cell 15:643-652

Martins-Rocha M, Cavalheiro GM, Matos-Rodrigues GE et al (2015) From gene targeting to genome editing: transgenic animal applications and beyond. An Acad Bras Cienc 87(2 Suppl):1323-1348

Maruyama T, Dougan SK, Truttmann MC et al (2015) Increasing the efficiency of precise genome editing with CRISPR-Cas9 by inhibition of nonhomologous end joinig. Nat Biotechnol 33:538-542

Menger L, Sledzinska A, Bergerhoff K et al (2016) TALEN-mediated inactivation of PD-1 in tumor-reactive lymphocytes promotes intratumoral T-cell persistence and rejection of established tumors. Cancer Res 76:2087-2093

Miller JC, Tan S, Qiao G et al (2011) A TALE nuclease architecture for efficient genome editing. Nat Biotechnol 29:143-148

Nemudryi AA, Valetdinova KR, Medvedev SP et al (2014) TALEN and CRISPR/Cas genome editing systems: tools of discovery. Acta Naturae 6:19-40

Nishimasu H, Ran FA, Hsu PD et al (2014) Crystal structure of Cas9 in complex with guide RNA and target DNA. Cell 156:935-949

Perez EE, Wang J, Miller JC et al (2008) Establishment of HIV-1 resistance in $\mathrm{CD}^{+} \mathrm{T}$ cells by genome editing using zinc-finger nucleases. Nat Biotechnol 26:808-816

Perica K, Varela JC, Oelke M et al (2015) Adoptive T cell immunotherapy for cancer. Rambam Maimonides Med J 6:e0004

Poirot L, Philip B, Schiffer-Mannioui C et al (2015) Multiplex genomeedited T-cell manufacturing platform for "off-the-shelf" adoptive T-cell immunotherapies. Cancer Res 75:3853-3864

Provasi E, Genovese P, Lombardo A et al (2012) Editing T cell specificity towards leukemia by zinc finger nucleases and lentiviral gene transfer. Nat Med 18:807-815

Qasim W, Zhan H, Samarasinghe S et al (2017) Molecular remission of infant B-ALL after infusion of universal TALEN gene-edited CAR T cells. Sci Transl Med 9 eaaj2013

Ran FA, Hsu PD, Lin CY et al (2013) Double nicking by RNA-guided CRISPR Cas9 for enhanced genome editing specificity. Cell 154:1380-1389

Schumann K, Lin S, Boyer E et al (2015) Generation of knock-in primary human T cells using Cas9 ribonucleoproteins. Proc Natl Acad Sci USA 112:10437-10442

Sharma R, Anguela XM, Doyon Y et al (2015) In vivo genome editing of the albumin locus as a platform for protein replacement therapy. Blood 126:1777-1784

Shen B, Zhang W, Zhang J et al (2014) Efficient genome modification by CRISPR-Cas9 nickase with minimal off-target effects. Nat Methods 11:399-402

Silva G, Poirot L, Galetto R et al (2011) Meganucleases and other tools for targeted genome engineering: perspectives and challenges for gene therapy. Curr Gene Ther 11:11-27

Stoddard BL (2011) Homing endonucleases: from microbial genetic invaders to reagents for targeted DNA modification. Structure 19:7-15

Su S, Hu B, Shao J et al (2016) CRISPR-Cas9 mediated efficient PD-1 disruption on human primary $\mathrm{T}$ cells from cancer patients. Sci Rep 6:20070

Su S, Zou Z, Chen F et al (2017) CRISPR-Cas9-mediated disruption of PD-1 on human T cells for adoptive cellular therapies of EBV positive gastric cancer. Oncoimmunology 6:e1249558

Tanenbaum ME, Gilbert LA, Qi LS et al (2014) A protein-tagging system for signal amplification in gene expression and fluorescence imaging. Cell 159:635-646

Tebas P, Stein D, Tang WW et al (2014) Gene editing of CCR5 in autologous CD4 T cells of persons infected with HIV. N Engl J Med 370:901-910 
Terns RM, Terns MP (2013) The RNA- and DNA-targeting CRISPRCas immune systems of Pyrococcus furiosus. Biochem Soc Trans 41:1416-1421

Tsai SQ, Wyvekens N, Khayter C et al (2014) Dimeric CRISPR RNAguided FokI nucleases for highly specific genome editing. Nat Biotechnol 32:569-576

Turtle CJ, Hanafi LA, Berger C et al (2016) Immunotherapy of nonHodgkin lymphoma with a defined ratio of $\mathrm{CD} 8^{+}$and $\mathrm{CD} 4^{+}$ CD19-specific chimeric antigen receptor-modified T cells. Sci Transl Med 8:355ra116

van Loenen MM, de Boer R, Amir AL et al (2010) Mixed T cell receptor dimers harbor potentially harmful neoreactivity. Proc Natl Acad Sci USA 107:10972-10977

van der Ploeg JR (2009) Analysis of CRISPR in Streptococcus mutans suggests frequent occurrence of acquired immunity against infection by M102-like bacteriophages. Microbiology 155(Pt 6):1966-1976

Wang J, DeClercq JJ, Hayward SB et al (2016) Highly efficient homology-driven genome editing in human $\mathrm{T}$ cells by combining zincfinger nuclease mRNA and AAV6 donor delivery. Nucleic Acids Res 44:e30

Yin H, Song CQ, Dorkin JR et al (2016) Therapeutic genome editing by combined viral and non-viral delivery of CRISPR system components in vivo. Nat Biotechnol 34:328-333

Zaslavskiy M, Bertonati C, Duchateau P et al (2014) Efficient design of meganucleases using a machine learning approach. BMC Bioinform 15:191 